\title{
Safe Longitudinal Platoons of Vehicles without Communication
}

\author{
Alexis Scheuer, Olivier Simonin and François Charpillet \\ LORIA / INRIA, MAIA team - Campus Scientifique - BP 239 \\ 54506 Vandœuvre-lès-Nancy Cedex FRANCE \\ [Alexis.Scheuer | Olivier.Simonin | Francois.Charpillet] dloria.fr
}

\begin{abstract}
This paper deals with the platooning problem that can be defined as the automatic following of a manned driven vehicle by a convoy of automatic ones. Different approaches have been proposed so far. Some require the localisation of each vehicle and a communication infrastructure, others called nearto-near approach only needs vehicle on-board sensors. However, to our knowledge, they do not provide any proof of non collision. We propose a novel near-to-near longitudinal platooning building a collision-free platooning whatever the number of vehicles. The model is derived from the study of the most dangerous interaction between two vehicles, i.e. considering the maximum acceptable acceleration when the previous vehicles brakes at maximum capacity. Collision avoidance of this model is proved. Finally, we show that this model can be combined to existing ones, keeping this collision-free property while allowing more various behaviors.
\end{abstract}

\section{INTRODUCTION}

Future urban transportation systems will include autonomous vehicles. The public transportation system we envision would be made up of a fleet of small electrical vehicles (often called CyberCar, $c f$. Figure 1) specifically designed for areas where car traffic must be severely restricted. This system could cover the urban areas with low demands or outside peak hours.

This new mode of public transport needs to be effective to ensure that there is always a balance between supplied and requested vehicles. A solution is to collect the vehicles distributed in areas with lower request. We imagine that a pilot could drive a leader vehicle, collecting vehicles that would automatically follow it to form a train without grip material.

In this paper we focus on the longitudinal platoon problem, by considering that vehicles are moving in a one dimensional space (along a line). As it has been shown that longitudinal and lateral control can be dealt separately [1], results obtained in this paper can be extended to the general platooning problem.

We consider the near-to-near approach as it does not require any infrastructure (GPS, wireless communication, etc.) which introduces additional complexity and unreliability in a platooning system. We think that such an approach is better suited to deal with the open challenge of ensuring that no collision can occur within platoons.

Several approaches have been explored to deal with nearto-near longitudinal platooning. Among the most typical

LORIA: Lab. Lorrain de Recherche en Informatique et ses Applications. INRIA: Institut National de Recherche en Informatique et Automatique.

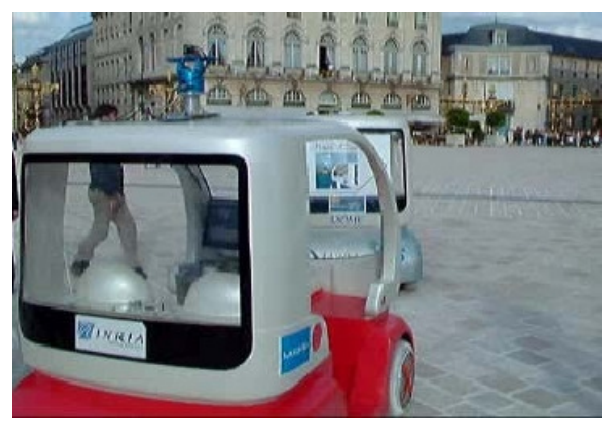

Fig. 1. CyberCar platoon with Cycabs (Mobivip exhibition, Nancy 2005).

models, we can quote the following references. Sheikholeslam and Desoer [5] proposed a longitudinal control based on linearization methods that focuses on stability of the convoy. Platoon stability is also tackled in [3] where a fixedgain PID control with gain scheduling is used. By contrast, a control mode based on a non-linear method with PID is proposed in [2], dealing with train stability but where collision accidents are assumed to be possible. As it was designed for CyberCars, we particularly examine in this paper the model introduced by Daviet and Parent [1] which relies on linear corrector with variable coefficients. Contrarily to these approaches we tackle the platooning problem by considering collision avoidance (and not stability of the platoon) as the main criteria to design safe platoons. We propose a novel approach building a collision-free platoon whatever the number of agents. The model is derived from the study of the most dangerous interaction between two vehicles, i.e. considering the maximum acceptable acceleration when the previous vehicles brakes at maximum capacity.

We do not study a particular model of autonomous vehicle, but we consider a generic vehicle/robot that may be controlled through its acceleration set point. We assume that each vehicle/robot owns a low level controller allowing to reach the acceleration set point. In order to make this assumption realistic we bound acceleration and speed values, and we consider a time delay to reach the set point.

The paper is organized as follows. section II defines the longitudinal platooning problem. Then, in section IV, we illustrate on the classical Daviet \& Parent approach that such a model do not ensure avoiding collisions. In section $\mathrm{V}$ we propose a collision-free platooning model, prove this property and show how to combine this approach with other platooning models. Then section VI discusses the robustness to perception uncertainty. Finally section VII concludes. 


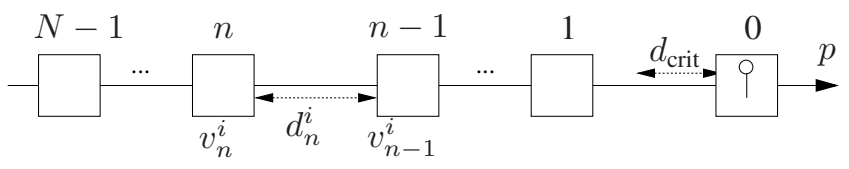

Fig. 2. Scheme of the problem and notations.

\section{The Platooning Problem}

\section{A. Hypotheses}

We consider a set of $N(\geqslant 2)$ vehicles forming a linear platoon. The first vehicle, numbered 0 , is driven by a human being. The others, numbered from 1 (following the leader) to $N-1$, are controlled by autonomous agents ( $c f$. Figure 2 ).

Motion of the considered vehicles is limited by fixed bounds on their velocity and acceleration, respectively called $v_{\min }, v_{\max }, a_{\min }$ and $a_{\max }$. We suppose that:

$$
0 \leqslant v_{\min }<v_{\max } \text { and } a_{\min }<0<a_{\max }
$$

We consider forward-only motions, with accelerations and decelerations. These limits can be due to traffic laws, passengers' comfort or any other constraints. They are generally far from engine's limits, to be sure that the low-level controller can always achieve the desired acceleration.

Leader's behavior is one of the problem input that greatly influence the platoon behavior. In this paper we simulate strong variations of velocity at different time steps, as presented in subsection III-B.

All the autonomous vehicles have a cyclic behaviour:

1) they perceive their environment,

2) they compute an acceleration set point,

3) they send this acceleration to the low-level controller.

We suppose each step is performed simultaneously by all the agents, i.e. they are synchronized.

We denote $\delta t$ the cycle duration, and $\tau$ the fixed delay (not known by agents) between perceptions and resulting actions. This delay includes durations for the perception, the acceleration computation and the transmission to low-level. We suppose $\tau<\delta t$.

At instant $i \delta t(i \geqslant 0)$, vehicle numbered $n(0<n<N)$ perceives:

- its velocity $v_{n}^{i}$

- its distance to previous (numbered $n-1$ ) vehicle $d_{n}^{i}$,

- the previous vehicle's velocity $v_{n-1}^{i}$.

Note that positions $p_{n}^{i}$ are not known by the vehicles. Contrary to infrastructure-based approaches we do not need to localise vehicles.

Actual sensors can estimate distances with such an accuracy that velocity of previous vehicle can be derived from it (using vehicle velocity). According to these perceptions, each vehicle decides which acceleration $a_{n}^{i}$ to apply to itself from instant $i \delta t+\tau$ to $(i+1) \delta t+\tau$.

Note: We present in section VI how errors in perceptions can be considered in the model.

\section{B. Non-Collision Property}

Collision avoidance is usually formulated as a strict inequality: distances should remain strictly positive. This induces that, at each time step, chosen accelerations should respect strict inequalities (to avoid future collisions): there is no optimal solution, as acceleration can be chosen as close as wanted to the forbidden limit.

We choose to define a critical distance $d_{\text {crit }}>0$, which may be as small as wanted, and consider that collision is avoided when distances between vehicles are greater than or equal to $d_{\text {crit }}: d_{n}^{i} \geq d_{\text {crit }}$. Non-collision constraint for accelerations thus admits an optimal (maximal) solution, which is used for our method (in section V).

\section{EXPERIMENTAL FRAMEWORK}

\section{A. Simulation Model}

In this paper, experiments are performed in simulation. The acceleration $a_{n}^{i}$ changes the position and velocity of the vehicle numbered $n$, according to elementary dynamic laws (straight motion with constant acceleration). However, bounds on velocities and accelerations make formulas more complex. The function move : $(p, v, a, t) \mapsto(p, v)$ computes the couple (position, velocity) obtained when acceleration $a$ is applied for a duration $t$, starting from position $p$ with a velocity $v$.

$$
\begin{aligned}
& \operatorname{move}(p, v, a, t)= \\
& \begin{cases}\left(v_{\min }, p+v_{\min } t-\left(v-v_{\min }\right)^{2} / a\right) & \text { if }\left(v+a t<v_{\min }\right) \\
\left(v_{\max }, p+v_{\max } t-\left(v_{\max }-v\right)^{2} / a\right) & \text { if }\left(v+a t>v_{\max }\right) \\
\left(v+a t, p+v t+a t^{2} / 2\right) & \text { otherwise }\end{cases}
\end{aligned}
$$

Moreover, as acceleration is only applied after a delay $\tau$, the couple (position, velocity) is computed in two steps. First, values are computed at instant $i \delta t+\tau:\left(p^{\prime}, v^{\prime}\right)=$ $\operatorname{move}\left(p_{n}^{i}, v_{n}^{i}, a_{n}^{i-1}, \tau\right)$, i.e. the previous acceleration $a_{n}^{i-1}$ is used before the delay $\left(a_{n}^{-1}=0\right)$. Then, new position and velocity are computed at instant $(i+1) \delta t$, as deduced from these values: $\left(p_{n}^{i+1}, v_{n}^{i+1}\right)=\operatorname{move}\left(p^{\prime}, v^{\prime}, a_{n}^{i}, \delta t-\tau\right)$.

\section{B. Experiments' Characterization}

As we consider several experiments in the following, the problem's data corresponding to each experiment will be given in the form of a vector, called a configuration. This vector contains $v_{\min }, v_{\max }, a_{\min }, a_{\max }$, the initial distances $d^{0}$ and velocities $v^{0}$, a minimum aimed distance $\Delta$ (used in most of the controllers), $\delta t, \tau$ and the leader's behavior. The minimum distance $d_{\text {crit }}$ and the number $N$ of vehicles are not included in the configuration, as they are constant for the experiments presented in this article: $d_{\text {crit }}$ is set to 0.05 meter, and 6 vehicles are considered (experiments with up to 12 vehicles gave similar results, with graphics harder to read - color graphics for any number of vehicles can be obtained with the on-line simulator, available at http: //www.loria.fr/ scheuer/Platoon).

The leader's behavior is given as a sequence of $k(k>0)$ couples, denoted $\left(t_{i}, v_{0}^{i}\right), 0 \leqslant i<k$. We suppose that the couples are ordered by increasing values of time, i.e. that $t_{i-1}<t_{i}(1 \leqslant i<k)$, with $t_{0}=0$. Considering that $t_{k}=$ $+\infty$, time axis can be cut in $k$ intervals $I_{i}=\left[t_{i}, t_{i+1}[\right.$. 
Each couple indicates the velocity the leader tries to reach, after the associated instant. At instant $t_{i}$ (leader reaction is not delayed by $\tau$ ), the leader acceleration $a_{0}^{i}$ is set to the optimal acceleration to reach velocity $v_{0}^{i}$ :

$$
a_{0}^{i}=a_{\min } \text { if } v_{0}^{i}<v_{0}\left(t_{i}\right) \text { and } a_{0}^{i}=a_{\max } \text { otherwise }
$$

Acceleration is maintained until $v$ reaches the desired velocity $v_{0}^{i}$ or $t$ reaches $t_{i+1}$, the end of interval $I_{i}$. Acceleration thus stops at $t_{i}^{*}=\min \left(t_{i+1}, t_{i}+\left(v_{0}^{i}-v_{0}\left(t_{i}\right)\right) / a_{0}^{i}\right)$. Note that velocity $v_{0}^{i}$ may not be reached, if $\left|v_{0}^{i}-v_{0}\left(t_{i}\right)\right|$ is too big with respect to $\left|t_{i+1}-t_{i}\right|$ and either $a_{\min }$ or $a_{\max }$.

The velocity profile of the leader is thus a piecewise linear curve as illustrated in Figure 3.

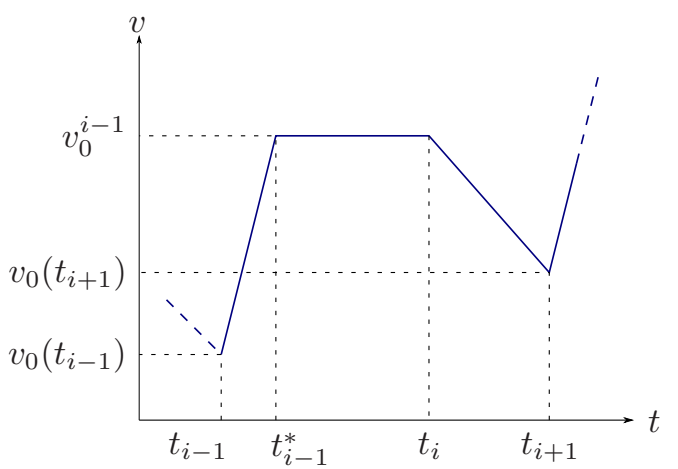

Fig. 3. Velocity profile for the leader.

\section{Study of Daviet and Parent Models}

Daviet and Parent [1] present a controller which compute acceleration $a_{n}^{i}$ as a function of $d_{n}^{i}, v_{n}^{i}$ and $v_{n-1}^{i}$, using the general formula

$$
a_{n}^{i}=\frac{\left(d_{n}^{i}-\Delta-h v_{n}^{i}\right) / C_{d}+v_{n-1}^{i}-v_{n}^{i}}{C_{v}}
$$

where $\Delta$ is the minimum aimed distance and $h$ is a "reaction delay" experimentally fixed to $0.35 \mathrm{~s}$ by authors. Two variants of the controller are proposed:

- constant coefficients controller uses $C_{d}=C_{v}=h$,

- variable coefficients controller uses $C_{v}=h$ and $C_{d}=$ $\max \left(h, v_{n}^{i} / a_{\max }\right)$.

Various experiments of these controllers has shown four global behaviors (detailed explanations can be found in [4]):

B1) accelerations and velocities quickly reach the intended values (without oscillation or long evolution);

B2) if the value $\Delta$ does not respect some constraints ${ }^{1}$, oscillations (and sometimes collisions) appear;

B3) the distance between vehicles may be quite smaller than the target distance at each cycle $(\Delta+h v)$, collisions may thus appear;

B4) the constant coefficients controller seems less stable than the variable coefficients one; oscillations are more frequently observed, and differences between real and aimed distances are greater.

\footnotetext{
${ }^{1}$ Typically, it has to be higher than a certain value, but these constraints are not explained in [1].
}
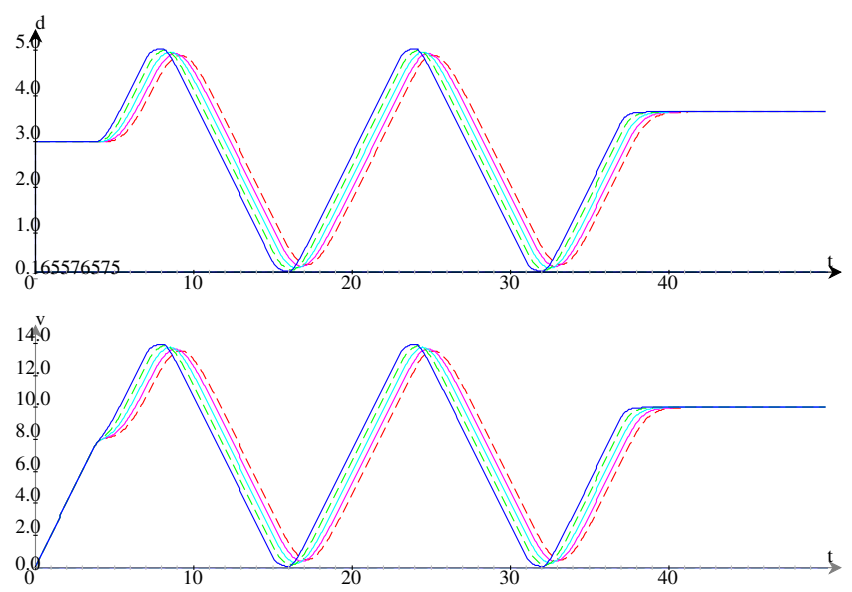

Fig. 4. Standard result using D \& P controllers.

Behavior B1 of the constant coefficients controller is illustrated in Figure 4, with $a_{\max }=-a_{\min }=2 \mathrm{~m} / \mathrm{s}^{2}, v_{\min }=0$ $\mathrm{m} / \mathrm{s}$ and $v_{\max }=14 \mathrm{~m} / \mathrm{s}(50.4 \mathrm{~km} / \mathrm{h}), d^{0}=3 \mathrm{~m}$ and $v^{0}=0$ $\mathrm{m} / \mathrm{s}, \delta t=0.01 \mathrm{~s}$ and $\tau=0.007 \mathrm{~s}, \Delta=0.15 \mathrm{~m}$, and the following behavior for the leader:

\begin{tabular}{l|c|c|c|c|c}
$t(\mathrm{~s})$ & 0 & 8 & 16 & 24 & 32 \\
\hline$v_{0}(\mathrm{~m} / \mathrm{s})$ & 14 & 0 & 14 & 0 & 10
\end{tabular}

On this figure and the following, one can see that distances and velocities only start to diverge after a delay. During this delay, distance between the vehicles is bigger than the target distance $\Delta+h v$ : all vehicles then have the same behavior.

Behavior B2 of the constant coefficients controller is illustrated in Figure 5 with $a_{\max }=-a_{\min }=0.5 \mathrm{~m} / \mathrm{s}^{2}$, $v_{\min }=0 \mathrm{~m} / \mathrm{s}$ and $v_{\max }=8 \mathrm{~m} / \mathrm{s}(28.8 \mathrm{~km} / \mathrm{h}), d^{0}=3 \mathrm{~m}$ and $v^{0}=0 \mathrm{~m} / \mathrm{s}, \delta t=0.01 \mathrm{~s}$ and $\tau=0.007 \mathrm{~s}, \Delta=0.17 \mathrm{~m}$, and the following behavior for the leader:

\begin{tabular}{l|c|c|c|c|c}
$t(\mathrm{~s})$ & 0 & 17.5 & 35 & 52.5 & 70 \\
\hline$v(\mathrm{~m} / \mathrm{s})$ & 8 & 0 & 8 & 0 & 6
\end{tabular}

In this figure, vehicle 1 is the only one to collide (with the leader), while all the agents have a final velocity oscillation. Setting $\Delta \geqslant 0.18 \mathrm{~m}$ seems necessary to switch to a normal behavior, i.e. without collision.

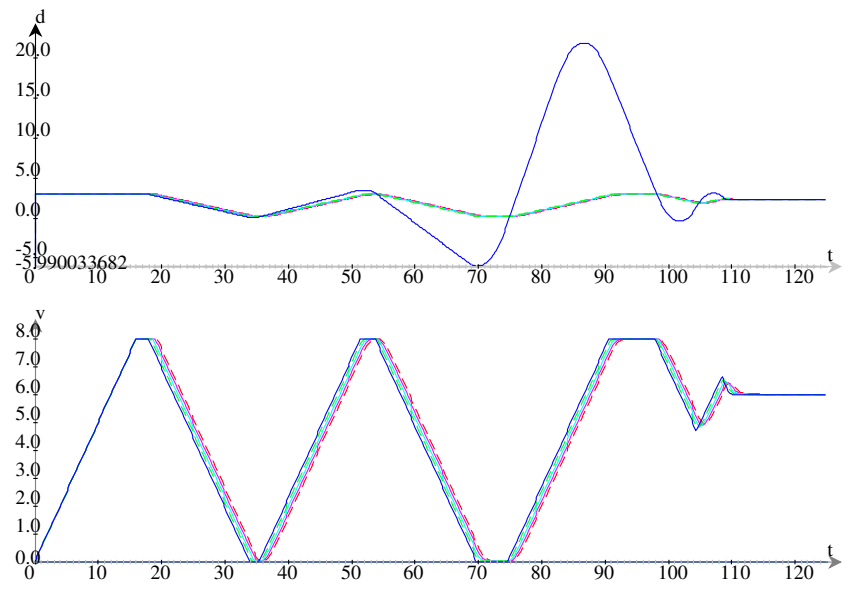

Fig. 5. D \& P constant coefficients controller in collision. 

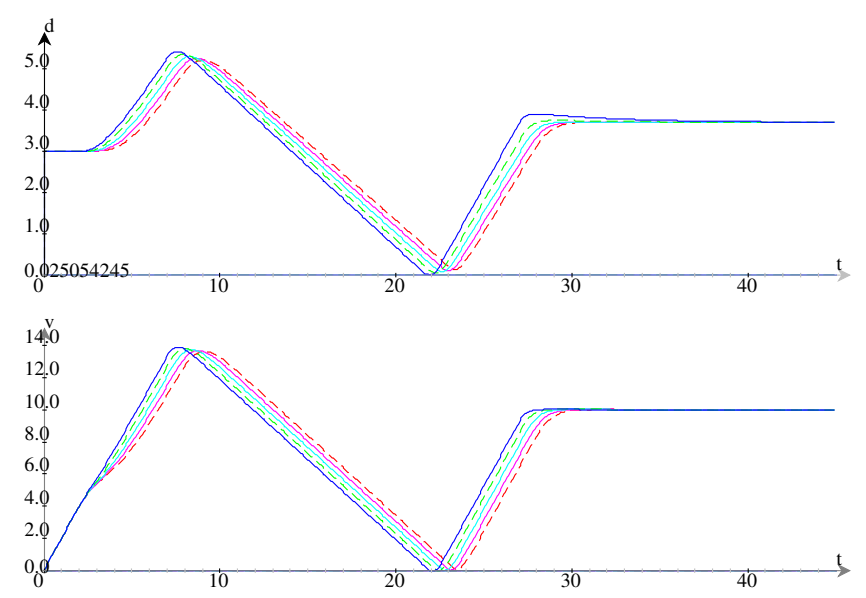

Fig. 6. D \& P variable coefficients controller in collision.

Behavior B3 of the variable coefficients controller is illustrated in Figure 6, with $a_{\min }=-1 \mathrm{~m} / \mathrm{s}^{2}, a_{\max }=2 \mathrm{~m} / \mathrm{s}^{2}$, $v_{\min }=0 \mathrm{~m} / \mathrm{s}$ and $v_{\max }=14 \mathrm{~m} / \mathrm{s}, d^{0}=3 \mathrm{~m}$ and $v^{0}=0 \mathrm{~m} / \mathrm{s}$, $\delta t=0.01 \mathrm{~s}$ and $\tau=0.007 \mathrm{~s}, \Delta=0.2 \mathrm{~m}$, and the following behavior for the leader:

\begin{tabular}{l|c|c|c}
$t(\mathrm{~s})$ & 0 & 7.5 & 22 \\
\hline$v(\mathrm{~m} / \mathrm{s})$ & 14 & 0 & 10
\end{tabular}

In Figure 6, minimum distance between the vehicles comes as low as $0.025 \mathrm{~m}$, which is much less than $\Delta(0.2 \mathrm{~m})$ and even less than $d_{\text {crit }}(0.05 \mathrm{~m})$ : collision occurs!

Oscillations (and often collisions) also appear when initial distances $d^{0}$ is smaller than the target distance associated to initial velocity $v^{0}$, i.e. when $d^{0}<\Delta+h v^{0}$. This constraint on initial values can be quite restrictive, due to the high value of $h(0.35 \mathrm{~s})$.

To avoid this constraint's effect, target distance can be reduced by changing the value of $h$ to a multiple of the time step $\delta t$ instead of using $0.35 \mathrm{~s}$. This however reduces the stability of controllers: oscillations appear more frequently. We will see in subsection V-B how this can be avoided.

To illustrate this behavior, we define a variant of the variable coefficients controller with $h=2 \delta t$. We call it fast $D \& P$. Figure 7 shows its behavior with the same

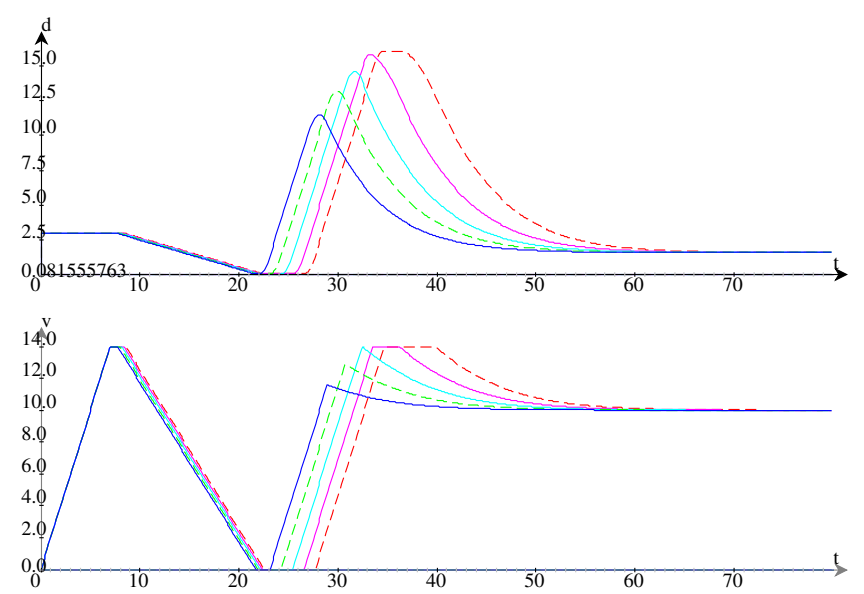

Fig. 7. Fast D \& P controller. configuration as for Figure 6, except $\Delta$ which is much higher (1.4 $\mathrm{m}$ instead of $0.2 \mathrm{~m}$ ). Difference between $\Delta$ and minimum distance obtained is now $0.6 \mathrm{~m}$ (1.4-0.8), instead of only $0.175(0.2-0.025)$. Moreover, the velocity do not reach efficiently the final aimed velocity: it takes more than 45 s to stabilize, while original D \& P controllers usually need less than $10 \mathrm{~s}$.

Finally, there is no analytical method to find the minimum aimed distance $\Delta$ so as to avoid collision for an infinite set of configurations (e.g., fixing some of the problem's data for example $v_{\min }, v_{\max }, a_{\min }, a_{\max }, \delta t$ and $\tau$ - and allowing any possible values for the others $-d^{0}, v^{0}$ and the leader's behavior).

\section{A Collision-Free Platooning}

We want to define a controller which ensures a safe behavior: collision with the others vehicles ${ }^{2}$ must always be avoided.

At first, we propose a controller verifying this property. We then show how it can be combined with other controllers in order to obtain different aimed distances, without loosing this property.

\section{A. Building a Controller Avoiding Collisions}

We want to ensure that vehicle $n$ can avoid collision with vehicle $n-1$ after time $i \delta t$, whatever the behavior of the previous one. Thus, we consider vehicle $n-1$ brakes at maximum capacity. A collision-free behavior exists if and only if maximum braking of vehicle $n$ allows to avoid collision. This is true when:

$$
d_{n}^{i} \geqslant d_{\text {crit }}+\max \left(0, \frac{v_{n}^{i}{ }^{2}-v_{n-1}^{i}{ }^{2}}{-2 a_{\min }}\right)
$$

where $a_{\min }$ is the minimum acceleration (or maximum deceleration) for all vehicles. Justification of this relation is presented in appendix VIII-A. Let $\delta d_{n}^{i}=d_{n}^{i}-d_{\text {crit }}+$ $\min \left(0,\left(v_{n}^{i^{2}}-v_{n-1}^{i}{ }^{2}\right) /\left(2 a_{\min }\right)\right)$. Inequality (1) can then be written simply $\delta d_{n}^{i} \geqslant 0$.

Analytical developments show this inequality (1) is verified when acceleration $a_{n}^{i}$ remains lower than or equal to

$$
\begin{aligned}
& a_{\lim }\left(d_{n}^{i}, v_{n}^{i}, v_{n-1}^{i}\right) \\
& =\min \left(a_{\min }+2 \frac{\tilde{d}_{n}^{i}-d_{\text {crit }}+\left(\tilde{v}_{n-1, n}^{i}-\tilde{v}_{n}^{i}\right) \delta t}{3 \delta t^{2}},\right. \\
& \frac{\sqrt{\left(\tilde{v}_{n}^{i}-a_{\min } \frac{\delta t}{2}\right)^{2}-2 a_{\min } \widetilde{\delta d}_{n}^{i}}-\left(\tilde{v}_{n}^{i}-\frac{3}{2} a_{\min } \delta t\right)}{\delta t}, \\
& \frac{\sqrt{\left(\tilde{v}_{n}^{i}+\left(a_{\max }-\frac{a_{\min }}{2}\right) \delta t\right)^{2}-2 a_{\min } \widetilde{D}_{n}^{i}}}{\delta t} \\
& \left.-\frac{\left(\tilde{v}_{n}^{i}+\left(a_{\max }-\frac{3}{2} a_{\min }\right) \delta t\right)}{\delta t}\right)
\end{aligned}
$$

\footnotetext{
${ }^{2}$ Other obstacles, like pedestrians, are not taken into account. We will see later that this is barely a problem, as distances between the vehicles are too small for an obstacle to interfere.
} 
where $\tilde{d}_{n}^{i}=d_{n}^{i}+\left(v_{n-1}^{i}-v_{n}^{i}\right) \delta t+\left(a_{\min }-a_{\max }\right) \delta t^{2} / 2$, $\tilde{v}_{n-1, n}^{i}=v_{n-1}^{i}+a_{\min } \delta t, \tilde{v}_{n}^{i}=v_{n}^{i}+a_{\max } \delta t, \widetilde{\delta d}_{n}^{i}=\tilde{d}_{n}^{i}-$ $d_{\text {crit }}+\left(\tilde{v}_{n}^{i 2}-\tilde{v}_{n-1, n}^{i}{ }^{2}\right) /\left(2 a_{\min }\right)$ and $\widetilde{D}_{n}^{i}=\max \left(0, \widetilde{\delta d}_{n}^{i}-\right.$ $\left.\left(a_{\max }-a_{\min }\right)\left(\tilde{v}_{n}^{i}+a_{\max } \delta t / 2\right) \delta t /\left(-a_{\min }\right)\right)+\left(a_{\max }-a_{\min }\right) \delta t^{2}$. In this formula, $\tilde{d}_{n}^{i}$ is a lower bound of $d_{n}^{i+1}, \tilde{v}_{n-1, n}^{i}$ a lower bound of $v_{n-1}^{i+1}, \tilde{v}_{n}^{i}$ an upper bound of $v_{n}^{i+1}, \widetilde{\delta d}_{n}^{i}$ a lower bound of $\delta d_{n}^{i+1}$, using the three previous ones, and $\widetilde{D}_{n}^{i}$ a lower bound of $\widetilde{\delta d}_{n}^{i+1}$.

\section{Theorem:}

With initial constraint $\widetilde{\delta d}_{n}^{0} \geqslant v_{n}^{0} \delta t(\forall n, 0 \leqslant n<N)^{3}$, if $a_{n}^{i} \leqslant a_{\lim }\left(d_{n}^{i}, v_{n}^{i}, v_{n-1}^{i}\right)(\forall n, 0<n<N$ and $\forall i \geqslant 0)$,

then collision cannot occur.

The main steps of the proof of this theorem are presented in appendix VIII-B (detailed proof can be found in [4]).

Note: Initial constraint is verified when vehicles are initially stopped, provided that initial distances are large enough. Experimental values remains close to $d_{\text {crit }}$.

The function $a_{\text {lim }}$ we just defined only provides an upper bound for the acceleration, in order to avoid collision. To fully define a controller, we still have to select which acceleration to take in the interval $\left[a_{\min }, a_{\mathrm{lim}}\right]$. This can be obtained, for example, by taking the minimum value between $a_{\lim }\left(d_{n}^{i}, v_{n}^{i}, v_{n-1}^{i}\right)$ and $a_{\max }$ : we call the resulting controller closest, as it tends to minimize distance between the vehicles.

Figure 8 gives an example of this controller's behavior, in the configuration of Figure 4. Distance is maintained to the minimum possible, except in the beginning where motion of the leading vehicle is the fastest possible (it is thus impossible to reduce the distance); otherwise, when the vehicle moves, the distance is slightly more than $d_{\text {crit }}$, due to reaction time, but remains less than $0.5 \mathrm{~m}$.
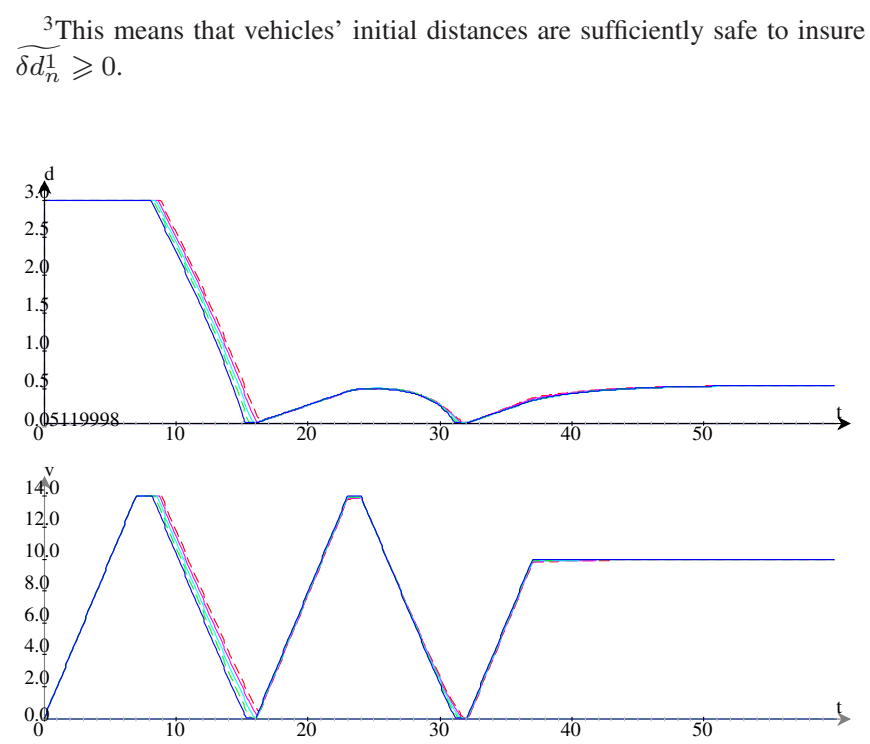

Fig. 8. Closest controller in motion.
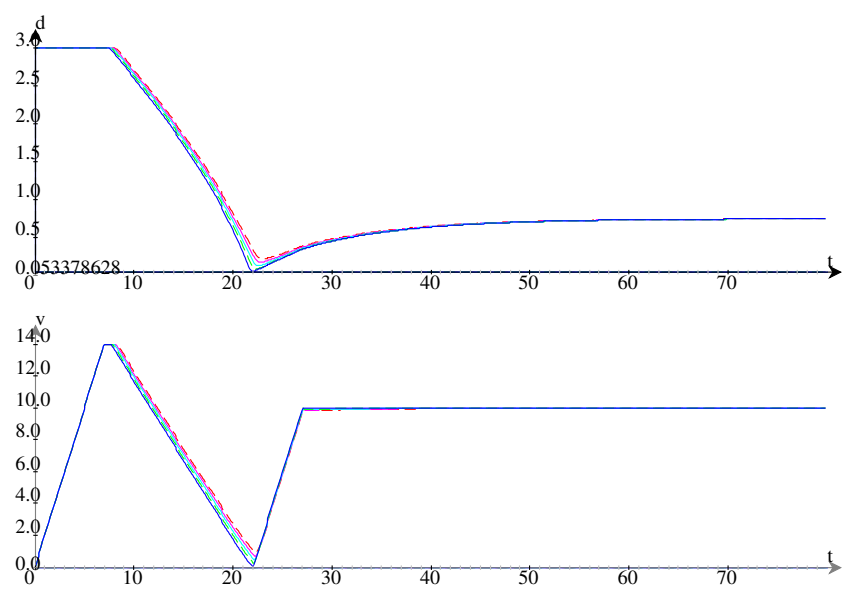

Fig. 9. Secure D \& P controller avoids collision.

\section{B. Combining with Other Controllers}

It is possible to have more various behaviors, by combining $a_{\text {lim }}$ function with another acceleration function $\gamma$ : selected acceleration is simply the minimum value between $a_{\lim }$ and $\gamma$. In that case, $a_{\lim }$ guarantees collision avoidance while $\gamma$ allows another behavior: it is thus possible to tend to a distance higher than the minimum required to avoid collision.

We call secure $D \& P$ the controller using as $\gamma$ function the fast D \& $\mathrm{P}$ controller, as defined at the end of previous section. Fig. 9 shows that this controller avoids collision even with a fixed ideal distance $\Delta$ of $0.05 \mathrm{~m}\left(=d_{\text {crit }}\right)$, in the configuration of Figure 6 and 7 where fast D \& P controller needed a $\Delta 28$ times higher $(1.4 \mathrm{~m})$.

\section{HANDLING ERRORS IN PERCEPtions}

As said at the end of section II, the problem presented in this article does not handle errors in perceptions, for simplicity reasons. These errors can be easily handled, once the algorithm understood, as they are bounded.

Let us call $\varepsilon_{d}(d)$ the bound on the error in distance perception: if a real distance $d$ is perceived as $d^{*},\left|d-d^{*}\right|$ is always lower or equal to $\varepsilon_{d}\left(d^{*}\right)$. We define similarly $\varepsilon_{v}$ and $\varepsilon_{v}^{f}: \varepsilon_{v}$ concerns the perception a vehicle has of its own velocity, while $\varepsilon_{v}^{f}$ concerns the perception of the leading vehicle's velocity.

As we need a lower bound of $\delta d_{n}^{i+2}$ to ensure collision avoidance, we need lower bounds of $d_{n}^{i+2}$ and $v_{n-1}^{i+2}$ and an upper bound of $v_{n}^{i+2}$. We then use lower bounds of $d_{n}^{i}$ and $v_{n-1}^{i}$ and an upper bound of $v_{n}^{i}$, computed from their perceived values (resp. $d_{n}^{i *}, v_{n-1}^{i}{ }^{*}$ and $v_{n}^{i *}$ ):

$$
\left\{\begin{aligned}
d_{n}^{i} & \geqslant d_{n}^{i *}-\varepsilon_{d}\left(d_{n}^{i *}\right) \\
v_{n-1}^{i} & \geqslant v_{n-1}^{i}{ }^{*}-\varepsilon_{v}^{f}\left(v_{n-1}^{i}{ }^{*}\right) \\
v_{n}^{i} & \leqslant v_{n}^{i *}+\varepsilon_{v}\left(v_{n}^{i *}\right)
\end{aligned}\right.
$$

These formulas can thus be used to replace exact values by perceptions with errors, as long as these errors can be bound (this is generally the case). 


\section{CONCLUSION}

We investigated in this paper the problem of collision avoidance in near-to-near longitudinal platoons of autonomous vehicles. We first considered the classical Daviet and Parent model, which is one of the rare approach to provide a generic controller separated from the low-level control. A systematic simulation-based analysis of this model has shown that non-collision and stability is not ensured in all cases. We then proposed an alternative model, building a collision-free platoon whatever the number of vehicles. It is derived from the study of the most dangerous interaction between two vehicles, i.e. considering the maximum acceptable acceleration when the previous vehicles brakes at maximum capacity. A sketch of the proof of the non-collision property is presented, showing how initial conditions can be fixed. Then, we shown how existing models can be combined to our approach, providing collision avoidance to more various behaviors. String stability of our approach has been experimentally observed, it still has to be theoretically studied. At last, these results have been extended to handle uncertainty in perception.

\section{APPENDICES}

\section{A. Distance to Avoid Collision}

In this section we justify that (1) ensures that vehicle $n$ can avoid collision with vehicle $n-1$ after time $i \delta t$, whatever the behavior of the previous one.

Considering worst case situation, distance between vehicles is monotone: its minimal value is obtained either at the beginning or at the end of the motion.

- If $v_{n-1}^{i} \geqslant v_{n}^{i}$, the distance between the vehicles grows; collision is then avoided iff $d_{n}^{i} \geqslant d_{\text {crit }}$.

- Otherwise (if $v_{n-1}^{i}<v_{n}^{i}$ ), this distance shrinks; times needed to stop are respectively $-v_{n}^{i} / a_{\min }$ and $-v_{n-1}^{i} / a_{\min }$ (where $a_{\min }<0$ ), distances to stop are thus $-v_{n}^{i^{2}} /\left(2 a_{\min }\right)$ and $-v_{n-1}^{i}{ }^{2} /\left(2 a_{\min }\right)$; collision is then avoided iff $d_{n}^{i} \geqslant d_{\text {crit }}-\left(v_{n}^{i}{ }^{2}-v_{n-1}^{i}{ }^{2}\right) /\left(2 a_{\min }\right)$.

\section{B. Sketch of the Non-Collision Proof}

Theorem in section $\mathrm{V}$ is proved by recurrence ${ }^{4}$, using initial conditions $\delta d_{n}^{0} \geqslant 0, \widetilde{\delta d}_{n}^{0} \geqslant 0$ and $\delta d_{n}^{1} \geqslant 0$, and the implication: $\forall n, 0 \leqslant n<N$ and $\forall i \geqslant 0$,

$$
\begin{aligned}
& {\widetilde{\delta d_{n}}}_{n} \geqslant 0 \text { and } a_{n}^{i} \leqslant a_{\lim }\left(d_{n}^{i}, v_{n}^{i}, v_{n-1}^{i}\right) \\
& \text { and, if } i>0, a_{n}^{i-1} \leqslant a_{\lim }\left(d_{n}^{i-1}, v_{n}^{i-1}, v_{n-1}^{i-1}\right) \\
& \Rightarrow{\widetilde{\delta d_{n}}}^{i+1} \geqslant 0 \text { and } \delta d_{n}^{i+2} \geqslant 0
\end{aligned}
$$

Initial condition of the theorem $\left(\widetilde{\delta d}_{n}^{0} \geqslant v_{n}^{0} \delta t\right)$ implies initial conditions of the recurrence (on $\delta d_{n}^{0}, \widetilde{\delta d_{n}}$ and $\delta d_{n}^{1}$ ). To get last one, we need:

- either $a_{n}^{0} \delta t^{2} / 2 \leqslant \tilde{d}_{n}^{0}-d_{\text {crit }}+a_{\max } \delta t^{2} / 2$ and $\delta d_{n}^{0}+\left(a_{n}^{0}-\right.$ $\left.a_{\min }\right)\left(v_{n}^{0}+a_{n}^{0} \delta t / 2\right) \delta t / a_{\min } \geqslant 0$ when $a_{n}^{0} \geqslant 0$;

- or $0 \leqslant \tilde{d}_{n}^{0}-d_{\text {crit }}+a_{\max } \delta t^{2} / 2$ and $\delta d_{n}^{0}-v_{n}^{0} \delta t \geqslant 0$ when $a_{n}^{0} \leqslant 0$.

${ }^{4}$ Detailed proof can be found in [4].
Developing $\widetilde{\delta d_{n}^{0}}$ easily leads to $\widetilde{\delta d_{n}^{0}} \leqslant \delta d_{n}^{0}$ and $\widetilde{\delta d_{n}^{0}} \leqslant \tilde{d}_{n}^{0}$ $d_{\text {crit }}$. Thus, both $\delta d_{n}^{0}-v_{n}^{0} \delta t$ and $\tilde{d}_{n}^{0}-d_{\text {crit }}$ are positive, and first, third and fourth needed conditions are true. Remaining condition is $f\left(a_{n}^{0}\right) \geqslant 0$, where $f(a)=\delta d_{n}^{0}+\left(a-a_{\min }\right)\left(v_{n}^{0}+\right.$ $a \delta t / 2) \delta t / a_{\min }$ is a quadratic function of negative main factor $\left(\delta t^{2} /\left(2 a_{\min }\right)\right)$. As both $f\left(a_{\min }\right)=\delta d_{n}^{0}$ and $f\left(a_{\max }\right)=\widetilde{\delta d}_{n}^{0}$ are positive, so is $f\left(a_{n}^{0}\right)$.

We thus proved $\delta d_{n}^{1} \geqslant 0, \delta d_{n}^{0} \geqslant 0$ and $\widetilde{\delta d}_{n}^{0} \geqslant 0$. Applying recurrently implication (3) directly proves the theorem. We still have to show how definition of $a_{\text {lim }}$ according to (2) proves implication (3).

A lower bound of $\delta d_{n}^{i+2}$ is computed ${ }^{5}$, in which $\tilde{d}_{n}^{i}$, $\tilde{v}_{n-1, n}^{i}, \tilde{v}_{n}^{i}$ and $\widetilde{\delta d}_{n}^{i}$ come respectively from $d_{n}, v_{n-1}, v_{n}$ and $\delta d_{n}^{i+1}$ computations. In this lower bound, $a_{n-1}^{k}, k \in$ $\{i-1, i, i+1\}$, is replaced by $a_{\min }$, as it is not known by vehicle $n, a_{n}^{i}$ is kept but $a_{n}^{i-1}$ and $a_{n}^{i+1}$, which are not known at instant $i \delta t$, are replaced by $a_{\max }$ (they have a negative factor).

As $\delta d_{n}^{i+2}$ contains a min function, $\delta d_{n}^{i+2} \geqslant 0$ implies two inequalities. The first one is linear with respect to $a_{n}^{i}$; it implies that $a_{n}^{i}$ should be lower than the first parameter of the min function in (2). The second one is quadratic with respect to $a_{n}^{i}$ : second term factor is negative, inequality is thus verified between the two roots; the smaller root is lower than $a_{\min }$, the higher root is the second parameter of the min function in (2).

A similar process is used to ensure $\widetilde{\delta d}_{n}^{i+1} \geqslant 0$, except that: - $a_{n}^{i}$ is replaced by $\max \left(a_{n}^{i-1}, a_{n}^{i}\right)$; thus, $\widetilde{\delta d}_{n}^{i+1} \geqslant 0$ leads to conditions similar to those found for $\delta d_{n}^{i+2} \geqslant 0$, but they constrain both $a_{n}^{i}$ and $a_{n}^{i-1}$;

- the lower bound on $\tilde{d}_{n}^{i+1}$ is higher than the one found for $d_{n}^{i+2}$; conditions on $a_{n}^{i}$ implying $\delta d_{n}^{i+2} \geqslant 0$ thus insure that those on $a_{n}^{i}$ for $\widetilde{\delta d}_{n}^{i+1} \geqslant 0$ are respected.

This leads to a quadratic constraint on $a_{n}^{i-1}$ which is respected when this acceleration remains lower than the third parameter of the min function in (2).

\section{ACKNOWLEDGEMENT}

This work was partially supported by $\mathrm{ANR}^{6}$ Tacos project and «Véhicule du futur » Cristal project ${ }^{7}$.

\section{REFERENCES}

[1] P. Daviet and M. Parent. Longitudinal and lateral servoing of vehicles in a platoon. In Proc. of the IEEE Int. Symp. on Intelligent Vehicles, pages 41-46, Tokyo (JP), September 1996.

[2] J. Hedrick, M. Tomizuka, and P. Varaiya. Control issues in automated highway systems. IEEE Control Systems Magazine, 14(6):21-32, 1994.

[3] P. Ioannou and $\mathrm{Z}$. Xu. Throttle and brake control systems for automatic vehicle following. IVHS Journal, 1(4):345-377, 1994.

[4] Alexis Scheuer, Olivier Simonin, and François Charpillet. A secure and stable behavior for long platoons of vehicles. Research report $\mathrm{n}^{\circ}$ 6741, Inst. Nat. de Recherche en Informatique et en Automatique, 2008. http://hal.inria.fr/inria-00342719.

[5] S. Sheikholeslam and C.A. Desoer. Longitudinal control of a platoon of vehicles with no communication of lead vehicle information: a system level study. IEEE Trans. on Vehicular Technology, 42(4):546-554, 1993.

\footnotetext{
${ }^{5}$ The maximum lower bound is chosen: this value can be reached

${ }^{6}$ French National Research Agency.

${ }^{7}$ Cf. http: / / www. vehiculedufutur. com.
} 